\title{
The influence of pre-radiation salivary flow rates and radiation dose on parotid salivary gland dysfunction in patients receiving radiotherapy for head and neck cancers
}

\begin{abstract}
Radiotherapy (RT) used for head and neck cancers causes permanent salivary gland dysfunction (SGD). Previous short-term studies have demonstrated that pre-RT salivary flow rates and the amount of radiation exposure to parotid glands influence the amount of RT-induced SGD. The purpose of this study was to determine which variables are related to the development of long-term post-RT SGD. Parotid flow rates (PFR) were assessed prior to and 1 year after completion of RT in spared parotid glands from 34 patients from 2 parotid-sparing protocols. The results reveal that spared PFR were not significantly higher 1 year post$R T$ in patients who had high pre-RT PFR, when compared with patients with low pre-RT PFR. However, patients who received higher doses of RT to spared parotid glands had lower PFR 1 year post-RT, compared with patients who had received lower doses of RT. These one-year findings suggest that high pre-RT PFR do not provide protection against RT-induced SGD. Conversely, reduced RT dosages to contralateral parotid glands are protective of PFR after completion of RT.
\end{abstract}

This paper won second prize in the 8th Annual Saul Kamen Scientific Report Contest. n 1995, there were an estimated 28,150 newly reported cases of oral and pharyngeal cancer in the United States, with an estimated 8,370 deaths from these cancers. ${ }^{1}$ Incidence rates of oral pharyngeal cancer increase with age, with over $95 \%$ of all oral cancers occurring in individuals aged $45+$ years. $^{2}$ Nearly $70 \%$ of these cancers occur in persons older than age $65 .^{2}$ The mortality rates for oral cancer also increase with age, and are high compared with other cancers, with an overall five-year survival rate of only $50 \%{ }^{3}$ Radiation therapy (RT) is a commonly used method of treatment for patients with head and neck cancers (H\&NC). The goal of RT is to eradicate the tumor without causing significant or permanent damage to the oral-pharyngeal tissues that are at low risk for cancer spread. ${ }^{4}$ Currently, many of the RT techniques used in the treatment of H\&NC have sideeffects detrimental to the oral cavity, including the loss of salivary gland function and a persistent complaint of a dry mouth (xerostomia).$^{5}$ As a consequence, patients suffer from oral discomfort and mucositis, difficulty chewing and swallowing, taste changes, dental caries, oral microbial changes, and chronic esophagitis. Severe reduction of salivary gland output not only results in a rapid deterioration in oral health but also has a negative impact on the quality of a person's life. ${ }^{6}$ Despite much research, there is still no effective treatment for radiation-induced salivary dysfunction (SGD)?

There is great inter-individual variation in the amount of SGD produced by RT. $^{8}$ Our previous studies have demonstrated that, through the use of three-dimensional treatment planning (3-DTP), radiation doses to contralateral parotid glands can be minimized, thereby reducing the amount of SGD in patients receiving either unilateral neck or partial bilateral neck RT for H\&NC. ${ }^{9-11}$ Currently, it is difficult to predict the extent of RT-induced SGD in a patient receiving radiation for $H \& N C$. Previous studies have identified some variables which may provide protection from RT-induced SGD. Two studies reported that patients with high preradiation salivary flow rates, independent of gland size, required larger doses of radiation to produce minimal flow rates. ${ }^{12,13}$ Other investigations have suggested that there is a dose-response relationship in parotid salivary flow rates in patients undergoing RT for H\&NC. ${ }^{12-14}$ In summary, based upon previous reports, it appears that three variables influence the amount of RT-induced SGD: preradiation unstimulated flow rates, pre-radiation stimulated flow rates, and radiation dose. However, most of these results are from short-term studies ( $<3$ months) which did not follow patients to ascertain long-term $(>1$ year) radiation changes. In addition, the assessment of radiation dose in all of these studies was performed 
by clinical estimation rather than by exact dosimetric methods. It is essential to know what predictors there are for long-term RT-induced SGD, since other investigators have reported that permanent SGD occurs after head and neck RT. ${ }^{5,12-14}$

The purpose of this investigation was to determine which variables are related to the development of SGD. If successful, results from this study could assist in predicting the salivaryrelated prognosis of a patient undergoing RT for H\&NC. It was hypothesized that patients with high preradiation parotid salivary flow rates would be more resistant to the deleterious effects of RT, and thus would be less likely to develop RT-induced SGD up to $1 \mathrm{yr}$ following completion of RT. It was also hypothesized that high RT doses to contralateral parotid glands would be associated with lower flow rates 1 yr post-RT.

\section{Materials and methods}

\section{Subjects}

Thirty-four subjects were examined from 2 parotid-sparing protocols currently under investigation at the University of Michigan (Table 1). ${ }^{9-11}$ Entrance criteria for patients included an histologically confirmed invasive cancer of the head and neck region (Table 2 ). Patients were excluded from this analysis if they had a poorly controlled systemic disease requiring concomitant medical intervention. Patients taking medications were also excluded from this analysis if they experienced changes during the treatment and follow-up period in drugs which are known to affect salivary function. ${ }^{15}$ Twenty patients with unilateral $H \& N C$ where the risk for contralateral neck node involvement was considered low $(<15 \%)$ received ipsilateral neck radiation. ${ }^{10}$ In 14 patients, where the tumor was more extensive and the risk for contralateral neck node involvement was considered high, bilateral neck radiation was used, while the contralateral parotid gland was at least partially spared RT. ${ }^{9,11}$
Table 1. Subjects.

\begin{tabular}{lccc}
\hline & $\begin{array}{c}\text { Ipsilateral Protocol } \\
(\mathrm{n}=20)\end{array}$ & $\begin{array}{c}\text { Bilateral Protocol } \\
(\mathrm{n}=14)\end{array}$ & p Value \\
\hline & & & \\
Age (yrs) (mean $\pm \mathrm{SD})$ & $60.6 \pm 2.5$ & $55.1 \pm 3.5$ & $\geq 0.05$ \\
Age range & $45-75$ & $20-82$ & \\
Gender & 11 males $/ 9$ females & 11 males $/ 3$ females & \\
& & & \\
\hline
\end{tabular}

Table 2. Cancer diagnosis and TNM stage.

\begin{tabular}{l}
\hline Tumor Type \\
Squamous cell carcinoma \\
Adenocystic carcinoma \\
Mucoepidermoid carcinoma \\
Neuroepithelial carcinoma \\
Neuroendocrine carcinoma \\
Malignant schwannoma \\
Large lymph cell carcinoma \\
Basal cell carcinoma
\end{tabular}

Tumor Location

Oral cavity

Hypopharynx

Larynx

2

$$
\mathrm{n}=34
$$

T Stage

$\begin{array}{ll}\mathrm{Tx}^{\mathrm{a}} & 2\end{array}$

$\mathrm{T}_{1}$

$\mathrm{T}_{2} \quad 12$

$\mathrm{T}_{3} \quad 5$

$\begin{array}{ll}\mathrm{T}_{4} & 7\end{array}$

Recurrent 1

$$
\mathrm{n}=34
$$

$\begin{array}{lc}\text { N Stage } & \\ \mathrm{N}_{0} & 15 \\ \mathrm{~N}_{1} & 5 \\ \mathrm{~N}_{2 \mathrm{a}} & 4 \\ \mathrm{~N}_{2 \mathrm{~b}} & 6 \\ \mathrm{~N}_{2 \mathrm{c}} & 1 \\ \mathrm{~N}_{3} & 2 \\ \text { Recurrent } & 1\end{array}$

$\mathrm{n}=34$ 
Table 3. Parotid salivary flow rates and radlation dose. ${ }^{a}$

\begin{tabular}{|c|c|c|c|c|c|c|}
\hline & \multicolumn{3}{|c|}{ Ipsilateral Protocol $(n=20)$} & \multicolumn{3}{|c|}{ Bilateral Protocol $(n=14)$} \\
\hline & Spared Gland & Treated Gland & $\mathrm{p}$ Value $^{\mathrm{c}}$ & Spared Gland & Treated Gland & $p$ Value $^{\mathrm{c}}$ \\
\hline \multicolumn{7}{|l|}{$\mathrm{UPFR}^{\mathrm{b}}$} \\
\hline Baseline & $0.07 \pm 0.06$ & $0.04 \pm 0.05$ & NS & $0.07 \pm 0.07$ & $0.10 \pm 0.11$ & NS \\
\hline $1 \mathrm{yr}$ post-RT & $0.06 \pm 0.08$ & 0 & 0.0001 & $0.03 \pm 0.06$ & 0 & 0.002 \\
\hline$p$ value ${ }^{d}$ & NS & 0.0003 & & 0.03 & 0.007 & \\
\hline \multicolumn{7}{|l|}{ SPFR $^{e}$} \\
\hline Baseline & $0.40 \pm 0.24$ & $0.24 \pm 0.47$ & NS & $0.38 \pm 0.21$ & $0.37 \pm 0.30$ & NS \\
\hline $1 \mathrm{yr}$ post-RT & $0.46 \pm 0.59^{f}$ & 0 & 0.02 & $0.22 \pm 0.23$ & 0 & 0.0009 \\
\hline$p$ value & NS & 0.001 & & 0.03 & 0.0005 & \\
\hline $\begin{array}{l}\text { Radiation dose } \\
\text { (Gy) }\end{array}$ & $3.6 \pm 2.0^{f}$ & $51.5 \pm 14.6$ & 0.0001 & $20.2 \pm 6.6$ & $57.1 \pm 5.6$ & 0.0001 \\
\hline
\end{tabular}

a Results expressed as mean $\pm \mathrm{SD}$.

b Unstimulated parotid flow rate $(\mathrm{mL} / \mathrm{min})$.

c Spared vs. treated glands.

d Baseline vs. $1 \mathrm{yr}$ post-RT.

e Stimulated parotid flow rate $(\mathrm{mL} / \mathrm{min})$.

$f \quad$ Ipsilateral spared vs. bilateral spared; $\mathrm{p}<0.05$.

\section{3-D radiation treatment planning (3-DTP)}

All patients underwent immobilization and full 3-DTP through the University of Michigan RadiationOncology planning system. ${ }^{16}$ 3-DTP and conformal dose delivery constitute a new therapeutic modality that conforms high-dose radiation volume to the shape of a tumor while minimizing the dose to tissue that is not at risk for containing cancer. Target volumes include the primary tumor mass and lymph node areas which contain metastases, along with lymph node groups that are at risk for microscopic extension. Treatment volumes for H\&NC and radiation dose to each parotid gland can be measured by the treatment planning computer program and cross-sectional CT images.

The goal of 3-DTP was full exclusion of the contralateral parotid gland (in the case of a lateralized tumor) or the gland at the side of the neck least involved with the tumor (in the case of bilateral neck involvement) from the primary beam while providing adequate volume coverage to the targets. If this was not possible,
3-DTP permitted the lowest dose possible to be administered to the contralateral parotid gland. All patients were treated with continuous conventional fractionation, and received 1.8-2.0 Gy fractions, 1 fraction per day, 5 fractions per week. All treatment was delivered with megavoltage energies by the linear acceleration or racetrack microtron.

\section{Parotid salivary flow measurements}

Bilateral parotid saliva samples were collected prior to RT (baseline) and 12 mos after the completion of RT. All salivary samples were collected between 8 a.m. and Noon to control for circadian variations in salivary gland function. ${ }^{17}$ Unstimulated parotid saliva (UPFR) was collected from both parotid gland orifices (Stensen's duct) with the use of a Carlson-

Crittenden cup for $2 \mathrm{~min}$ as described previously. ${ }^{18}$ Salivary flow was stimulated (SPFR) by $2 \%$ citric acid swabbed on the dorso-lateral surfaces of the tongue at 30-second intervals for $2 \mathrm{~min}$ for equilibration. This was followed by a two-minute collection period during which gustatory stimulation was maintained. Following collection, the volumes of all saliva samples were determined gravimetrically by means of an analytical balance, assuming a specific gravity of 1.0 .

\section{Statistical analysis}

Paired tests were used for comparisons of UPFR and SPFR between treated and spared glands for each collection period. Comparisons were also performed for UPFR and SPFR between baseline and one-year postcompletion of RT for the treated and spared glands. A paired $t$ test was used where mean values were assumed to have a normal distribution, and a two-sample robust analysis was used where normality was not a plausible assumption. ${ }^{19}$ Direct comparisons (paired $t$ tests) were used for the evaluation of differences in the amounts of radiation delivered to the spared and treated glands.

The 3 variables investigated were: (1) baseline unstimulated parotid flow rates (UPFR) from spared glands, (2) baseline stimulated parotid flow rates (SPFR) from 
spared glands, and (3) mean radiation dose to spared parotid glands. Patients in the ipsilateral and bilateral radiation protocols were placed into separate groups. For the first 2 variables at baseline, patients in each of the 2 radiation protocols were analyzed separately by being divided into two subgroups: highest $1 / 2$ and lowest $1 / 2$. For the third variable, patients in both protocols were combined, since there were unequal numbers of subjects in the ipsilateral and bilateral groups. This analysis for radiation dose compared subjects in the highest $1 / 4$ and lowest $1 / 4$. Within each group, PFR at baseline and $1 \mathrm{yr}$ post-RT were compared by paired $t$ tests. One year post-radiation spared UPFR and SPPR were compared by Student's $t$ tests between the highest $1 / 2$ and lowest $1 / 2$ group for each of the first two variables, and between the highest $1 / 4$ and lowest $1 / 4$ group for the third variable. A criterion of $p$ $<0.05$ was accepted for significance in all statistical tests.

\section{Results}

This paper describes the results from 34 subjects in two parotid-sparing protocols treated with the use of 3DTP. Patients in both the ipsilateral $(p=0.0001)$ and bilateral $(p=0.0001)$ protocols received significantly greater radiation to treated parotid glands compared with spared glands (Table 3). On average, radiation exposure to the spared gland was less than one-tenth (ipsilateral protocol) and almost one-third (bilateral protocol) the total amount of radiation delivered to the treated glands. During the one-year period following RT, there were no cases of cancer recurrence in all 34 patients in the regions spared radiotherapy.

UPFR and SPFR from treated and spared glands at baseline and $1 \mathrm{yr}$ post-RT are shown in Table 3. For patients in both the ipsilateral and bilateral protocols, UPFR and SPFR in the treated glands were significantly lower $1 \mathrm{yr}$ after the completion of RT compared with baseline. One year post-RT UPFR from spared glands in patients in the ipsilateral protocol

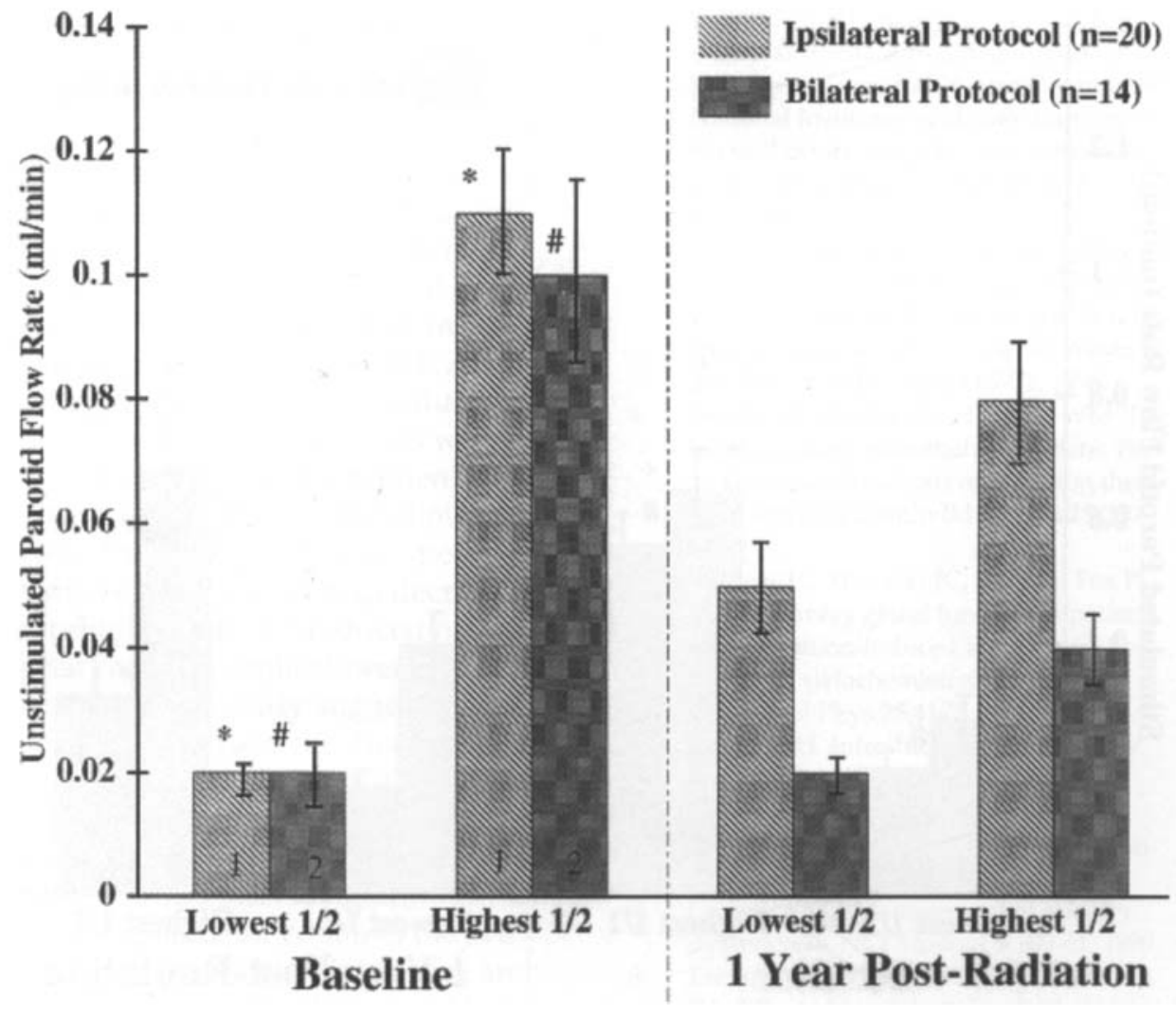

Fig 1. Unstimulated parotid flow rates (UPFR) at baseline (pre-RT) and 1 yr after the completion of RT in ipsilateral and bilateral RT protocols. Patients were divided into lowest $1 / 2$ and highest $1 / 2$ groups based on pre-RT (baseline) UPFR. Bars with the same number indicate patients receiving similar amounts of radiation, while those with different numbers indicate patients receiving significantly different amounts of radiation. Groups with the same symbols $(*, \#)$ indicate significantly different $(p<0.05)$ flow rates. Results are expressed as mean \pm SEM.

were statistically indistinguishable compared with baseline ( $\mathrm{p}>0.05)$; however, 1 yr post-RT UPFR from spared glands were significantly lower in patients from the bilateral protocol $(p=0.03)$. Similarly, SPFR 1 yr post-RT in the spared glands from the ipsilateral protocol were statistically indistinguishable compared with baseline ( $p>0.05)$, and were significantly lower in patients from the bilateral protocol $(\mathrm{p}=0.03)$. Furthermore, in the ipsilateral and bilateral protocols, UPFR and SPFR were not significantly different at baseline $(p>0.05)$ between the treated and spared glands, but were significantly different 1 yr after RT (ipsilateral protocol-UPFR $\mathrm{p}=0.0001$, SPFR $\mathrm{p}=0.02$; bilateral protocolUPFR $p=0.002$, SPFR $p=0.0009$ ).

To determine which variables pro- vide protection against $\mathrm{RT}$-induced SGD, patients in the ipsilateral and bilateral protocols were divided separately into two subgroups-highest $1 / 2$ and lowest $1 / 2$ - based on baseline UPFR and SPFR values from spared glands. For unstimulated saliva, flow rates between the highest $1 / 2$ and lowest $1 / 2$ groups were not significantly different 1 yr after the completion of RT for patients in the ipsilateral and bilateral protocols (Fig. 1). For stimulated saliva, flow rates were also not significantly different between the highest $1 / 2$ and lowest $1 / 2$ groups 1 yr after the completion of RT for patients in both ipsilateral and bilateral protocols (Fig. 2).

To determine the influence of radiation doses on parotid function, subjects from both the ipsilateral and bilateral protocols were combined 


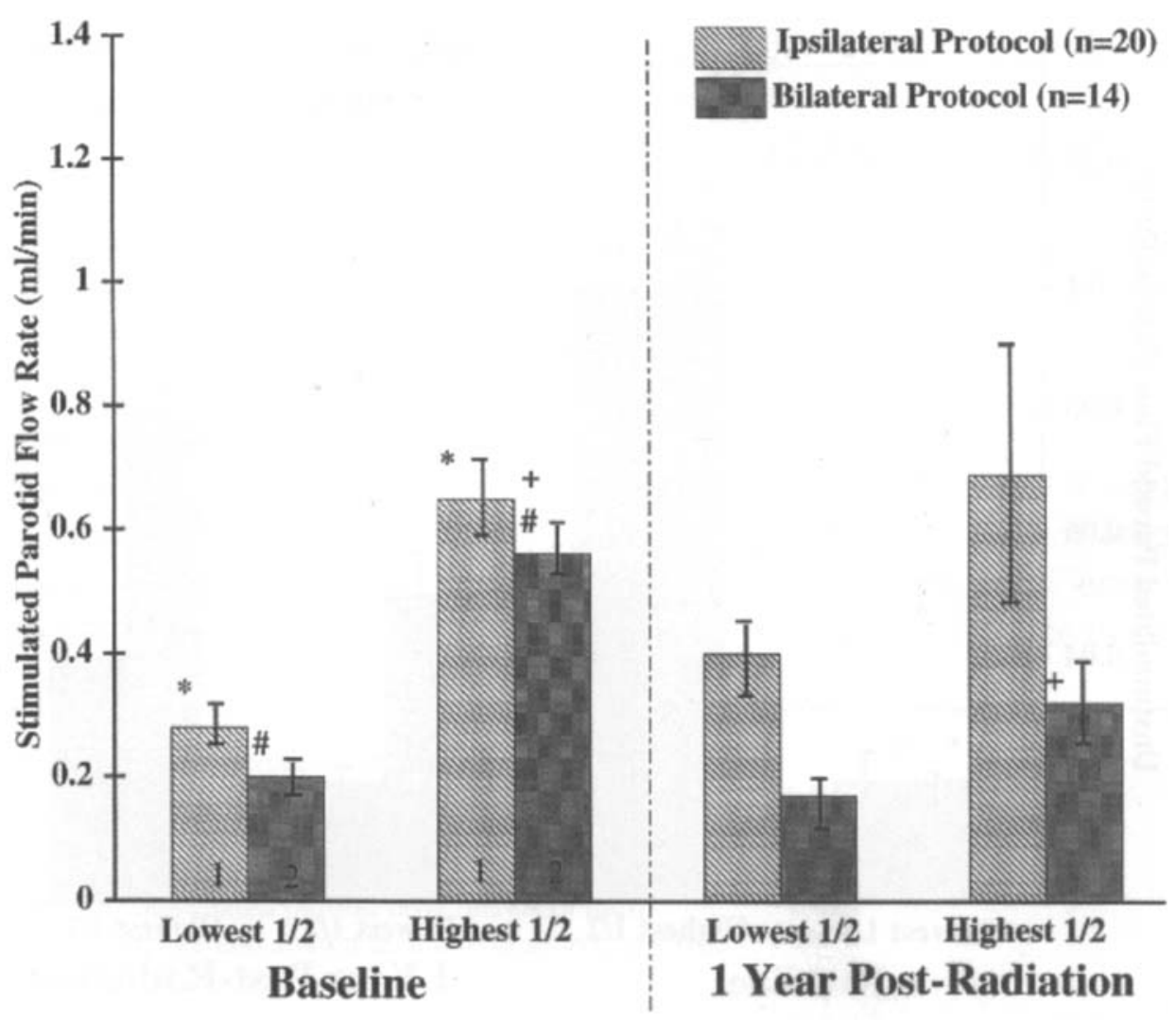

Fig 2. Stimulated parotid flow rates (SPFR) at baseline (pre-RT) and $1 \mathrm{yr}$ after the completion of RT in ipsilateral and bilateral RT protocols. Patients were divided into lowest 1/2 and highest 1/2 groups based on pre-RT (baseline) SPFR. Bars with the same number indicate patients receiving similar amounts of radiation, while those with different numbers indicate patients receiving significantly different amounts of radiation. Groups with the same symbols $\left({ }^{*}, \#,+\right)$ indicate significantly different $(p<$ $0.05)$ flow rates. Results are expressed as mean \pm SEM.

Table 4. Radiation dose. ${ }^{\mathrm{a}}$

\begin{tabular}{|c|c|c|c|}
\hline & $\begin{array}{c}\text { Radiation Dose } \\
\text { Lowest } 1 / 4(n=8)\end{array}$ & $\begin{array}{c}\text { Radiation Dose } \\
\text { Highest } 1 / 4(\mathrm{n}=8)\end{array}$ & $\begin{array}{l}\text { p Value } \\
\text { Lowest vs. Highest }\end{array}$ \\
\hline \multicolumn{4}{|l|}{$\mathrm{UPFR}^{\mathrm{b}}$} \\
\hline Radiation dose (Gy) & $2.0 \pm 0.8$ & $22.0 \pm 10.0$ & 0.0001 \\
\hline Baseline flow (mL/min) & $0.09 \pm 0.02$ & $0.09 \pm 0.03$ & $\mathrm{NS}^{\mathrm{c}}$ \\
\hline 1-yr flow $(\mathrm{mL} / \mathrm{min})$ & $0.07 \pm 0.02$ & $0.03 \pm 0.03$ & 0.06 \\
\hline $\mathrm{p}$ value: Baseline $v s .1 \mathrm{yr}$ & NS & 0.03 & \\
\hline \multicolumn{4}{|l|}{ SPFR $^{d}$} \\
\hline Radiation dose (Gy) & $2.0 \pm 0.8$ & $22.0 \pm 10.0$ & 0.001 \\
\hline Baseline flow $(\mathrm{mL} / \mathrm{min})$ & $0.52 \pm 0.12$ & $0.35 \pm 0.20$ & NS \\
\hline $1-\mathrm{yr}$ flow $(\mathrm{mL} / \mathrm{min})$ & $0.60 \pm 0.15$ & $0.16 \pm 0.04$ & 0.01 \\
\hline p value: Baseline vs. $1 \mathrm{yr}$ & NS & 0.02 & \\
\hline
\end{tabular}

Results expressed as mean \pm SEM

Unstimulated parotid flow rate.

Not statistically significant.

Stimulated parotid flow rate. and placed into 2 groups based on radiation dose to spared parotid glands: highest $1 / 4$ and lowest $1 / 4$ (Table 4). UPFR and SPFR were not significantly different at baseline. However, $1 \mathrm{yr}$ after the completion of $\mathrm{RT}$, flow rates were higher in the lowest $1 / 4$ radiation group compared with the highest $1 / 4$ radiation group (UPFR $p=0.06$; SPFR $p=0.01$ ). When comparisons were performed between UPFR and SPFR at baseline and $1 \mathrm{yr}$ following the completion of RT within each radiation group, flow rates were not significantly different in the lowest $1 / 4$ radiation group (UPFR $\mathrm{p}>0.05$; SPFR $\mathrm{p}>0.05$ ), but were significantly lower in the highest $1 / 4$ radiation group (UPFR $p=$ 0.03 ; SPFR $\mathrm{p}=0.02$ ).

\section{Discussion}

Head and neck RT for oral and pharyngeal cancers has a deleterious effect on salivary gland function. $4,5,12,14,16$ There is a dose-response relationship between the amount of radiation delivered to oral tissues and the damage that eventually occurs. ${ }^{5,12,14}$ Radiation doses of 2100 to $4000 \mathrm{cGy}$ to tissues of the head and neck region have been shown to cause significant damage to salivary glands. ${ }^{12}$ The results of our previous studies have shown that through the use of 3-DTP, radiation dosages to contralateral parotid glands can be minimized. ${ }^{9-11}$ Patients requiring unilateral radiation received a mean of $93 \%$ less radiation to spared glands, while patients receiving bilateral radiation received a mean of $65 \%$ less radiation to spared glands compared with treated parotid glands (Table 3). This reduction in radiation dose resulted in a partial preservation of contralateral parotid gland function, while nearly all output was lost in treated parotid glands.

The majority of studies which have examined factors that influence the amount and severity of RTinduced SGD have been short-term investigations ( $<3$ mos). Several short-term studies reported that people with higher initial salivary flow rates required larger doses of radia- 
tion to produce salivary hypofunction. ${ }^{8,13}$ These studies suggested that the dose-response relationship between SGD and RT is not linear, but is exponential, with the most severe SGD occurring during the early treatment periods of RT. ${ }^{8,13}$ It is possible that high baseline salivary flow rates may protect against SGD in the early stages of head and neck $\mathrm{RT},{ }^{8,13}$ however, after the accumulation of therapeutic doses of RT (usually $\geq 60 \mathrm{~Gy}$ ), all parotid gland function is destroyed 6 to 12 mos following the completion of RT. ${ }^{20}$

In the present study, patients were divided into two groups (highest $1 / 2$ and lowest $1 / 2$, based on baseline UPFR and SPFR) to determine if high initial parotid gland flow rates protected against RT-induced SGD. The results demonstrate that although patients in both groups had significantly different flow rates at baseline and received similar amounts of radiation, UPFR and SPFR were not significantly different 1 yr after the completion of RT. In addition, in patients who received bilateral neck $R T$ and had high initial salivary flow rates, there was a trend for both UPFR and SPFR to be lower $1 \mathrm{yr}$ after the completion of RT (Figs. 1, 2). Therefore, the one-year results from the present study do not provide any evidence that high baseline UPFR or SPFR provide protection against $\mathrm{RT}$-induced SGD.

Alternatively, the one-year results of this study demonstrate that a significant relationship does exist between the amount of RT delivered to spared parotid glands and the extent of SGD. Previous studies have examined UPFR and SPFR and have showed that patients in the lowest radiation dose quartile ( $<50$ Gy) had significantly higher salivary flow rates compared with those in the highest dose quartile (> $68 \mathrm{~Gy}){ }^{5}$ In the present study, patients who underwent ipsilateral neck RT (mean dose of 3.6 Gy to contralateral parotid glands) had higher UPFR and SPFR 1 yr after RT, compared with patients who received bilateral neck RT (mean dosage of 20.2 Gy to contralateral parotid glands). In addition, UPFR and SPFR from spared glands in the bilateral protocol $1 \mathrm{yr}$ after RT were significantly lower compared with baseline, while patients who received ipsilateral RT did not experience significant changes in parotid output from contralateral spared glands.

These results have clinical implications for the care of head and neck cancer patients receiving RT. A significant predictor for RT-induced SGD could identify patients who may be vulnerable to the detrimental sideeffects of RT. This would allow clinicians the ability to initiate special preventive measures to help decrease morbidity from RT-induced oral and pharyngeal complications. ${ }^{6,21}$ The results of this study suggest that the amount of radiation delivered to contralateral parotid glands should be minimized as much as possible, depending on the cancer stage, so that some parotid salivary gland function can be spared. Since saliva plays a central role in maintaining oral and pharyngeal health, this would help preserve oral function and a person's quality of life in patients following the completion of RT.

This study was funded by the University of Michigan School of Dentistry (Department of Oral Medicine/Pathology/Surgery), the University of Michigan Medical Center (Department of Radiation Oncology and the Department of Hospital Dentistry), Oral Health America-America's Fund for Dental Health Grant \#94-32, the University of Michigan School of Dentistry Student Research Program (NIDR Training Grant DE07101), and an American Association for Dental Research Student Research Fellowship (to Mr. D'Hondt). The authors thank the American Society for Geriatric Dentistry for awarding Mr. D'Hondt Second Place in the 8th Annual Saul Kamen Scientific Report Contest for this research project. The authors also acknowledge the assistance of Mr. Brad Henson and Dr. Aparna Madhava (University of Michigan School of Dentistry) for data collection and analysis.

Mr. D'Hondt and Dr. Ship are in the Department of Oral Medicine, Pathology, Surgery, University of Michigan School of Dentistry, 1011 N. University, Room 2012, Ann Arbor, MI 48109-1078. Dr. Eisbruch is in the Department of Radiation Oncology, University of Michigan Medical Center, Ann Arbor, MI. Correspondence should be addressed to Dr. Ship.

1. Wingo PA, Tong TA, Bolden S. Cancer statistics. CA Cancer J Clin 45:8-30, 1995.

2. US Department of Health and Human Services. Centers for Disease Control and National Institutes of Health. Cancers of the oral cavity and pharynx: A statistics review monograph 1973-1987. Bethesda, MD, 1991.

3. Nyman J, Mercke C, Lindstrom J. Prognostic factors for local control and survival of cancer of the oral tongue. A retrospective analysis of 230 cases in Western Sweden. Acta Oncol 32:667-73, 1993.

4. Semba SE, Mealey BL, Hallmon WM. The head and neck radiotherapy patient: Part 1 - Oral manifestations of radiation therapy. Compend Contin Educ Dent 15:25061, 1994.

5. Valdez IH, Atkinson JC, Ship JA, Fox PC. Major salivary gland function in patients with radiation-induced xerostomia: flow rates and sialochemistry. Int J Radiat Oncol Biol Phys 25:41-7, 1993.

6. Whelton $\mathrm{H}$. Introduction: The anatomy and physiology of salivary glands. In: Saliva and oral health. Edgar WM, O'Mullane DM, editors. London: British Dental Association, pp. 1-9, 1996.

7. Greenspan D. Management of salivary dysfunction. NCI Monogr 9:159-61, 1990.

8. Eneroth $\mathrm{CM}$, Henrickson CO, Jakobsson PA. Effect of fractionated radiotherapy on salivary gland function. Cancer 30:1147$54,1972$.

9. Eisbruch AE, Ship JA, Martel MK, Ten Haken RK, Marsh LH, Wolf GT, et al. Parotid gland sparing in patients undergoing bilateral head and neck irradiation: techniques and early results. Int J Radiat Oncol Biol Phys 36:469-80, 1996.

10. Jones R, Takeuchi T, Eisbruch A, D'Hondt EG, Hazuka M, Ship JA. Ipsilateral parotid sparing study in head and neck cancer patients receiving radiation therapy: 1 year results. Oral Surg Oral Med Oral Pathol Oral Radiol Endo 81:642-8, 1996.

11. Ship JA, Eisbruch AE, D'Hondt EG, Jones RA. Parotid sparing study in head \& neck cancer patients receiving bilateral radiation therapy: 1 year results. J Dent Res 76:807-13, 1997.

12. Mira JG, Wescott WB, Starcke EN, Shannon IL. Some factors influencing salivary function when treating with radiotherapy. Int J Radiat Oncol Biol Phys 7:535-41, 1981.

13. Wescott W, Mira JQ, Starcke EN, Shannon IL, Thornby JI. Alteration in whole saliva flow rate induced by fractionated radiotherapy. Am J Roentgenol 130:145-9, 1978.

14. Marks JE, Davis CC, Gottsman VL, Purdy JE, Lee $F$. The effects of radiation on parotid salivary function. Int J Oncol Biol Phys 7:1013-9, 1981.

15. Sreebny LM, Schwartz SS. A reference guide to drugs and dry mouth. Gerodontology 5:75-99, 1986.

16. Hazuka MB, Martel MK, Marsh L, Lichter AS, Wolf GT. Preservation of parotid 
function after external beam irradiation in head and neck cancer patients: a feasibility study using 3-dimensional (3-D) treatment planning. Int $\mathrm{J}$ Radiat Oncol Biol Phys 27:731-7, 1993.

17. Dawes $C$. Rhythms in salivary flow rate and composition. Int J Chronobiol 2:253$79,1974$.
18. Baum BJ. Evaluation of stimulated parotid saliva flow rates in different age groups. J Dent Res 60:1292-6, 1991.

19. DuMouchel W. A data analysis advisor. Technical report. Cambridge, MA: BBN Software Products Corporation, 1987.

20. Makkonen TA, Nordman E. Estimation of the long-term salivary gland damage induced by radiotherapy. Acta Oncologica 26:307-12, 1987.

21. Parsons JT. Time-dose-volume relation in radiation therapy. In: Management of head and neck cancer. A multidisciplinary approach. Million RR, Cassini NJ, edifors. Philadelphia, PA: Lippincott $\mathrm{Co}$., pp. 173-243, 1994 